The most significant results allowed us to conclude that the large majority of adolescents recognize AIDS as a normal disease. The most commonly reported method of transmission was sexual contact with a person already infected. 52.54\% of the interviewees mentioned having taken preventive measures. Although $42.01 \%$ of them have done nothing.

The majority considered that condoms were the safest method and showed an intention of use in all circumstances.

\title{
P032 - Look at me - who do you see? What are the impacts of Facebook profiles on the emotional wellbeing of young people?
}

\author{
C. Mackrell (United Kingdom)ํㅜㄹ C. Hall (United Kingdom) ${ }^{1}$
}

${ }^{1}$ School of Health Sciences, University of Nottingham

Theme: Ethical issues: dignity and humanity.

Keywords: Dignity, emotional wellbeing, Facebook, social media, young people, social media.

Social media is a critical form of communication for young people. For those requiring nursing care, and physically removed from usual communities, impressions of social media may be particularly influential.

To explore the impact Facebook has on wellbeing this study evaluated young people's perceptions of Facebook-style profiles using Likert scales and open questions. A total of 40 students situated within Erikson's (1968) 'ego identity versus role confusion' stage of development participated.

Findings identified that these young people were influenced by profile content viewed in a Facebook style, and that negative social comparisons they frequently made reduced their self-esteem. This has implications for young people and for CYP nurses.

To offer best practice for young people, nurses must understand the importance of social media and yet acknowledge potential risks. New student nurses of a similar age should be professionally aware to ensure respect and dignity in judgments which may affect patient care.

Erikson E (1968) Identity: Youth and Crisis. Norton, New York NY. 